\title{
Gauge and Lorentz Anomalies in the Pure Spinor Formulation of the Heterotic Superstring
}

\author{
Vladimir Pershin* \\ Instituto de Ciências Exatas, \\ Universidade Federal de Itajubá \\ Av. BPS 1303, 37500-903 \\ Itajubá-MG, Brazil \\ E-mail: pershin@unifei.edu.br
}

The $\mathrm{N}=1$ supergravity/super-Yang-Mills theory in $\mathrm{D}=10$ has gauge and Lorentz anomalies which can be cancelled by Green-Schwarz mechanism. Unfortunately, the new terms that this mechanism introduces to the theory action break supersymmetry and until now it was not possible to find their supersymmetrization. The covariant quantization of superstring in supergravity/super-YangMills background could resolve this problem. We use the Berkovits formulation of the heterotic superstring in background fields to calculate the gauge and Lorentz anomalies of the theory in terms of superfields at the 1 loop level and find explicit form of the counterterms and redefinitions of the antisymmetric superfield that cancel the anomalies. These results can be used to construct a supersymmetric generalization of the Green-Schwarz mechanism.

Fourth International Winter Conference on Mathematical Methods in Physics

09 - 13 August 2004

Centro Brasileiro de Pesquisas Fisicas (CBPF/MCT), Rio de Janeiro, Brazil

\footnotetext{
${ }^{*}$ Speaker.
} 
Absence of local anomalies is an important restriction on any consistent physical theory (see e.g. [1]). In the case of the superstrings and their low energy effective field theory approximations the cancellation of the gauge and gravitational anomalies [2] leads to existence of the five perturbatively consistent models. The IIA theory does not contain chiral fermions and so is free of anomalies, in the IIB theory the anomalies cancel due to a specific field content [1]. Another three perturbative superstring models are Type I, Het $S O(32)$ and Het $E_{8} \times E_{8}$ and in the low energy limit they are described by the $\mathrm{N}=1 \mathrm{D}=10$ supergravity coupled to $\mathrm{D}=10$ super-Yang-Mills. Anomaly cancellation in this theory is provided by the Green-Schwarz mechanism [2].

Let us remind some details of this mechanism. The physical fields of the D=10 SYM are the gauge field $A_{m}^{I}$ and the Weyl gaugino $\lambda_{\alpha}^{I}$. Bosonic part of the action is

$$
\int d^{10} x \operatorname{tr}\left(F^{2}\right), \quad F=d A+A^{2} .
$$

The fields of the $\mathrm{D}=10 \mathrm{~N}=1$ supergravity are the graviton $e_{m}^{a}$, the antisymmetric tensor $b_{m n}$, the dilaton $\phi$, the right gravitino $\psi_{m}^{\alpha}$ and the left dilatino $\chi_{m}^{\alpha}$. The bosonic part of the action is

$$
\int d^{10} x \sqrt{-G} e^{-2 \phi}\left(R+4 \partial_{m} \phi \partial^{m} \phi-\frac{1}{2} H^{2}\right), \quad H=d b .
$$

A subtle point is that in order to couple these two theories together in a supersymmetric way it is necessary to include the terms like $\sim \alpha^{\prime} A_{m} F_{n k} H^{m n k}$ which break the gauge invariance $\delta_{\text {gauge }} A_{m}^{I}=$ $\partial_{m} \varepsilon^{I}$. To restore the gauge invariance in the coupled theory one should make the $b$-field transform as [3]:

$$
\delta_{\text {gauge }} b_{m n}=\alpha^{\prime} A_{[m}^{I} \partial_{n]} \varepsilon^{I} .
$$

The new (gauge invariant) field strength is

$$
\tilde{H}=d b-\alpha^{\prime} \omega_{3}^{Y M}, \quad \omega_{3}^{Y M}=\operatorname{tr}\left(A d A+\frac{2}{3} A A A\right) .
$$

As a result, the bosonic part of the action becomes

$$
\int d^{10} x \sqrt{-G} e^{-2 \phi}\left(R+4 \partial_{m} \phi \partial^{m} \phi-\frac{1}{2} \tilde{H}^{2}+\alpha^{\prime} \operatorname{tr}\left(F^{2}\right)\right) .
$$

The theory contains chiral fermions and so is potentially anomalous. The Green-Schwarz mechanism [2] cancels both gauge and gravitational (Lorentz) anomalies and consists in the following steps:

1. choosing specific gauge groups $S O(32)$ or $E_{8} \times E_{8}$

2. introducing the Lorentz transformation for the $b$-field

$$
\delta_{\text {Lorentz }} b=\alpha^{\prime} \operatorname{tr}\left(\Sigma d \omega_{1}\right)
$$

$\left(\omega_{1}=\omega_{m}{ }^{a b} d x^{m}\right.$ is the spin connection for the vielbein $\left.e_{m}^{a}\right)$ and redefining its field strength by the Lorentz-Chern-Simons form

$$
\tilde{H}_{3}=d b-\alpha^{\prime} \omega_{3}^{Y M}-\alpha^{\prime} \omega_{3}^{L}, \quad \omega_{3}^{L}=\operatorname{tr}\left(\omega_{1} d \omega_{1}+\frac{2}{3} \omega_{1}^{3}\right)
$$


3. adding to the action some other terms of higher orders in derivatives like, for example,

$$
\int d^{10} x\left(\alpha^{\prime}\right)^{3} b\left(\operatorname{tr} F^{4}+\operatorname{tr} R^{4}+\operatorname{tr} R^{2} \operatorname{tr} F^{2}\right)+\ldots
$$

The problem is that all these new terms as well as the $b$-field redefinitions break supersymmetry. From the other hand, the theory is believed to have a supersymmetric extension because it is a low-energy limit of the supersymmetric string theories. So soon after the discovery of the Green-Schwarz mechanism several groups started looking for its supersymmetric generalization.

The first group of these efforts (se e.g. [4]) concentrated on possible deformations of the classical action and the supersymmetry transformations in the component form. These deformations were calculated up to the order $\sim R^{4}, \sim F^{4}$, for example, the bosonic part of the action was shown to be

$$
\begin{gathered}
S \sim \int \sqrt{-G} e^{-2 \phi}\left\{R+4(\nabla \phi)^{2}-\tilde{H}^{2}+\alpha^{\prime}\left(\operatorname{tr} F^{2}-\operatorname{tr} R^{2}\right)\right. \\
\left.+\left(\alpha^{\prime}\right)^{3} t_{8}\left(\operatorname{tr} F^{2}-\operatorname{tr} R^{2}\right)^{2}\right\}
\end{gathered}
$$

where $t_{8}$ is a numerical 8-rank tensor defined in [2]. However, the component calculations become extremely difficult in higher orders and usually tell nothing about general structure of the deformed theory. In particular, it is not clear from the component analysis whether it is possible to find a finite set of extra terms to make the theory supersymemtric or supersymmetrization is possible only perturbatively, order by order in $\alpha^{\prime}$.

Superfield approach is more suitable for such general questions. In the case of $D=10$ supersymmetric theories superfield description is known only on-shell, in other words, we have only superfields equations of motion without action principle.

$\mathrm{D}=10 \mathrm{~N}=1$ curved superspace is parametrized by coordinates $Z^{M}=\left(x^{m}, \theta^{\mu}\right), m=0, \ldots, 9, \mu=$ $1, \ldots, 16$. The superfields of the $\mathrm{N}=1$ supergravity are the supervielbein 1-forms $E^{A}=d Z^{M} E_{M}{ }^{A}(Z)$, the superconnection 1-forms $\omega_{A}^{B}=d Z^{M} \omega_{M A}^{B}(Z)$, and the 2-form $B=\frac{1}{2} E^{C} E^{A} B_{A C}$. Their field strengths are respectively the torsion $T^{A}=D E^{A}=\frac{1}{2} E^{C} E^{B} T_{B C^{A}}$, the curvature $R_{A}{ }^{B}=d \omega_{A}{ }^{B}+$ $\omega_{A}{ }^{C} \omega_{C}{ }^{B}=\frac{1}{2} E^{D} E^{C} R_{C D A}{ }^{B}$, and $H=d B=\frac{1}{3 !} E^{C} E^{B} E^{A} H_{A B C}$, with the corresponding Bianchi identities

$$
D T^{A}-E^{B} R_{B}{ }^{A}=0, \quad d H=0 .
$$

These superfields have too many components to accomodate the degrees of freedom of the theory and one should impose some constraints to receive the physical content of SUGRA. One possible choice is [5]

$$
T_{\alpha \beta}^{a}=2 \gamma_{\alpha \beta}^{a}, T_{\alpha \beta}^{\gamma}=0, T_{\alpha a}^{b}=0, T_{a \alpha}{ }^{\beta}=\left(\gamma_{a} \psi\right)_{\alpha}{ }^{\beta}, H_{\alpha \beta \gamma}=0 .
$$

Conditions of consistency of the constraints with the Bianchi identities define the on-shell theory.

The theory can be coupled consistently with the super-Yang-Mills by changing the Bianchi identity

$$
d H=c_{1} \operatorname{tr} F^{2}, \quad H=d B+c_{1} \omega_{3}^{Y M}
$$


and leaving the constraints unchanged [6]. A problem arises when one tries to accomodate the Green-Schwarz mechanism into this scheme. The Bianchi identity in this case should have become

$$
d H=c_{1} \operatorname{tr} F^{2}+c_{2} \operatorname{tr} R^{2}, \quad H=d B+c_{1} \omega_{3}^{Y M}+c_{2} \omega_{3}^{L},
$$

but this is inconsistent with the constraints above [6]. It means that the constraints should also be deformed but a full consistent form of this deformation has never been found (for a recent review of this activity see [7] and the references therein).

From the string theory point of view the need of changing of the definition of $b$-field strength can be seen as the condition of anomaly cancellation in the corresponding 2-dimensional $\sigma$-model [8].

A simplified example is provided by the heterotic string in background gauge field:

$$
S=\int d^{2} z\left(\frac{1}{2} \bar{\psi}_{A} \partial \bar{\psi}_{A}+A_{m}^{I}(x) \partial x^{m} \bar{J}^{I}\right)
$$

where $\bar{J}^{I}=\frac{1}{2} \bar{\psi}^{T} \tau^{I} \bar{\psi}$ is the current of the chiral fermions $\bar{\psi}_{A}$ and $\left[\tau^{I}, \tau^{J}\right]=f^{I J K} \tau^{K}$.

At the classical level the theory is invariant under the transformations

$$
\delta \bar{\psi}_{A}=\Lambda^{I}(x) \tau_{A B}^{I} \bar{\psi}_{B}, \quad \delta A_{m}^{I}=-\partial_{m} \Lambda^{I}(x)-f^{I J K} A_{m}^{K} \Lambda^{J}(x)
$$

Strictly speaking, they are not gauge transformations from the 2-dimensional point of view. They act on the space of theories defined for different $A_{m}(x)$ and not on the fields of the given theory.

Integrating out the fermions

$$
\Gamma[A]=<e^{-\int A \partial x \bar{J}}>
$$

one can see that the effective action is not invariant

$$
\delta \Gamma[A]=-\int d^{2} z \partial_{m} \Lambda^{I} A_{n}^{I} \partial x^{n} \bar{\partial} x^{m} .
$$

The symmetric part in $(m n)$ can be cancelled by the local counterterm

$$
\delta\left(\frac{1}{2} \int d^{2} z A_{n}^{I} A_{m}^{I} \partial x^{n} \bar{\partial} x^{m}\right)=\int d^{2} z \partial_{(n} \Lambda^{I} A_{m)}^{I} \partial x^{n} \bar{\partial} x^{m}
$$

and so does not represent anomaly. To cancel the antisymmetric part we should consider the $b$-field with the action

$$
\int d^{2} z b_{m n}(x) \partial x^{m} \bar{\partial} x^{n}
$$

and define its gauge variation as

$$
\delta b_{m n}=\partial_{[m} \Lambda^{I} A_{n]}^{I} .
$$

Then $\delta \Gamma[A]=0$.

To see how the other ingredients of the GS mechanism appear from the string side one needs to quantize the full superstring theory in SUGRA/SYM background. But until recently covariant supersymmetric quantization of the superstring was not possible. The Ramond-Neveu-Schwarz formulation of the superstring is covariant but space-time supersymemtry is not explicit in it, and this leads to difficulties in the case of fermionic and RR background fields. The Green-Schwarz 
superstring has explicit supersymmetry but cannot be quantized covariantly, due to the problem of separation of first/second class constraints.

Recently Berkovits [9] made an important step toward covariant quantization of the superstring proposing that the theory ghosts should the structure of pure spinors. This cannot yet be seen directly from the first principles but the proposal has already passed a number of consistency checks and lead to some new results [10].

In the case of pure spinor formulation of the heterotic superstring the world-sheet variables consist in the superspace coordinates $\left(x^{m}, \theta^{\alpha}, p_{\alpha}\right)$, the left-moving pure spinor ghosts $\lambda^{\alpha}\left(\lambda^{\alpha} \lambda^{\beta} \gamma_{\alpha \beta}^{m}=\right.$ 0 ), their conjugate momenta $w_{\alpha}$, the gauge right-moving currents $\bar{J}^{I}$ and the right-moving Virasoro ghosts $(\bar{b}, \bar{c})$.

The action is quadratic and so represents a free CFT:

$$
S=\frac{1}{4 \pi \alpha^{\prime}} \int d^{2} z\left(\frac{1}{2} \partial x^{m} \bar{\partial} x_{m}+p_{\alpha} \bar{\partial} \theta^{\alpha}+\bar{b} \partial \bar{c}\right)+S_{\lambda}+S_{J}
$$

$S_{\lambda}$ and $S_{J}$ are the actions for $\lambda^{\alpha}$ and $\vec{J}^{I}$. Physical states of the superstring are defined by the cohomology of the left- and right-moving BRST operators

$$
Q=\oint \lambda^{\alpha} d_{\alpha}, \quad \bar{Q}=\oint(\bar{c} \bar{T}+\bar{c} \bar{\partial} \bar{c} \bar{b})
$$

where $d_{\alpha}$ are the fermionic constraints of the GS formalism, and $T$ is the Virasoro constraint.

The generalization of the theory for the curved SUGRA/SYM background is given by the following action [11]:

$$
\begin{aligned}
S= & \frac{1}{2 \pi \alpha^{\prime}} \int d^{2} z\left\{\frac{1}{2}\left(G_{M N}(Z)+B_{M N}(Z)\right) \partial Z^{M} \bar{\partial} Z^{N}+E_{M}^{\alpha}(Z) d_{\alpha} \bar{\partial} Z^{M}\right. \\
& +\Omega_{M \alpha}^{\beta}(Z) \lambda^{\alpha} w_{\beta} \bar{\partial} Z^{M}+A_{M}^{I}(Z) \partial Z^{M} \bar{J}^{I}+W^{\alpha I} d_{\alpha} \bar{J}^{I} \\
& \left.+\frac{1}{2} U_{\alpha}^{I} \beta(Z) \lambda^{\alpha} w_{\beta} \bar{J}^{I}+\frac{1}{2} \alpha^{\prime} \Phi(Z) r+\bar{b} \partial \bar{c}\right\}+S_{\lambda}+S_{J} .
\end{aligned}
$$

Here $M=(m, \mu)$ are curved superspace indices, $Z^{M}=\left(x^{m}, \theta^{\mu}\right), A=(a, \alpha)$ are tangent superspace indices, and $r$ is the world-sheet curvature.

It is already known that the requirement of classical BRST invariance of this theory leads to the on-shell constraints of $N=1$ SUGRA and SYM [11]. Quantum BRST invariance is expected to modify these constraints by $\alpha^{\prime}$ corrections and to lead to a supersymmetric version of the GS mechanism.

Recently Chandia and Vallilo [12] constructed covariant background expansion in the theory under consideration using the technique analogous to that used previously in the $\mathrm{D}=4$ string calculations [13]. They showed that the theory is UV-finite at 1-loop if the SUGRA/SYM superfields are on-shell.

Having in mind possible supersymmetrization of the Green-Schwarz mechanism we constructed the 1-loop effective action and found counterterms and redefinitions of the superfield transformations that cancel the gauge and Lorentz anomalies from the worldsheet point of view [14]. 
The 1-loop effective action has a non-local structure

$$
\Gamma=\int d^{2} z d^{2} w\left(\frac{f(z, w)}{(z-w)^{2}}+\frac{g(\bar{z}, \bar{w})}{(\bar{z}-\bar{w})^{2}}\right)
$$

but to calculate anomalies one has to look only for local terms in its variation under the combined gauge/Lorentz transformation. Such local terms in the variation appear when we apply the derivatives from $\delta A_{A}^{I}=-\nabla_{A} \Lambda^{I}, \delta \Omega_{A a b}=-\nabla_{A} \Sigma_{a b}$ to $1 /(z-w)^{2}, 1 /(\bar{z}-\bar{w})^{2}$ and use

$$
\bar{\partial}_{\bar{w}} \frac{1}{(z-w)^{2}}=2 \pi \partial_{z} \delta^{(2)}(z-w), \quad \partial_{w} \frac{1}{(\bar{z}-\bar{w})^{2}}=2 \pi \bar{\partial}_{\bar{z}} \delta^{(2)}(z-w) .
$$

The answer is [14]

$$
\delta \Gamma=\frac{1}{2 \pi} \int d^{2} z\left\{-\frac{1}{2}\left(\bar{\partial} \Omega_{a b}+\bar{\partial} \alpha_{a b}\right) \Sigma^{a b}-\left(\bar{\partial} A^{I}+\bar{\partial} \beta^{I}\right) \Lambda^{I}\right\}
$$

where

$$
\begin{aligned}
& \Omega_{a b}=\Pi^{A} \Omega_{A a b}, \quad \alpha_{a b}=-\frac{1}{2}\left(d_{\alpha} T_{a b}{ }^{\alpha}+\lambda^{\alpha} w_{\beta} R_{a b \alpha}{ }^{\beta}-\Pi^{A} T_{A a b}\right), \\
& A^{I}=\Pi^{A} A_{A}^{I}, \quad \beta^{I}=d_{\alpha} W^{I \alpha}+\lambda^{\alpha} w_{\beta} U_{\alpha}^{I}{ }^{\beta} .
\end{aligned}
$$

By adding the counterterm

$$
S_{C}=\frac{1}{2 \pi} \int d^{2} z \bar{A}^{I}\left(\frac{1}{2} A^{I}+\beta^{I}\right)+\bar{\Omega}_{a b}\left(\frac{1}{4} \Omega^{a b}-\alpha^{a b}\right),
$$

we can eliminate the covariant part of $\delta \Gamma$.

The non-covariant (connections) terms are eliminated if we define

$$
\delta B_{N M}(Z)=\alpha^{\prime}\left(\Lambda^{I} \partial_{[N} A_{M]}^{I}+\frac{1}{2} \Sigma^{a b} \partial_{[N} \Omega_{M] a b}\right),
$$

which is a superfield generalization of the $b$-field redefinition.

Thus cancellation of gauge/Lorentz anomalies in the world-sheet $\sigma$-model corresponding to heterotic superstring in SUGRA/SYM background is realized in a manifestly supersymmetric way. The next step should be construction of 1-loop BRST transformations and then using the requirement of quantum BRST invariance should lead to supersymmetrization of the GS mechanism.

\section{References}

[1] L. Alvarez-Gaumé and E. Witten, Gravitational anomalies, Nucl. Phys. B234 (1983) 269.

[2] M. B. Green and J. H. Schwarz, Anomaly cancellations in supersymmetric d $=10$ gauge theory and superstring theory, Phys. Lett. B151 (1984) 117.

[3] E. Bergshoeff, M. de Roo, B. de Wit and P. van Nieuwenhuizen, Ten-dimensional Maxwell-Einstein supergravity, its currents, and the issue of its auxiliary fields, Nucl. Phys. B195 (1982) 97; G. F. Chapline and N. S. Manton, Unification of Yang-Mills theory and supergravity in ten dimensions, Phys. Lett. B120 (1983) 105. 
[4] E. Bergshoeff and M. de Roo, The quartic effective action of the heterotic string and supersymmetry, Nucl. Phys. $\mathbf{3 3 2 8}$ (1989) 439; M. de Roo, H. Suelmann and A. Wiedemann, The supersymmetric effective action of the heterotic string in ten dimension, Nucl. Phys. B405 (1993) 326 [hep-th/9210099].

[5] E. Witten, Twistor-like transform in ten dimensions, Nucl. Phys. B266 (1986) 245.

[6] J. J. Atick, A. Dhar and B. Ratra, Superspace formulation of ten-dimensional $N=1$ supergravity coupled to $N=1$ super-Yang-Mills theory, Phys. Rev. D33 (1986) 2824.

[7] S. J. Gates, A. Kiss and W. Merrell, Dynamical equations from a first-order perturbative superspace formulation of 10D, $N=1$ string-corrected supergravity (I), hep-th/0409104.

[8] C. M. Hull and E. Witten, Supersymmetric sigma models and the heterotic string, Phys. Lett. B160 (1985) 398.

[9] N. Berkovits, Super-Poincare covariant quantization of the superstring, JHEP 0004 (2000) 018 [hep-th/0001035]; ICTP lectures on covariant quantization of the superstring, hep-th/0209059.

[10] N. Berkovits and V. Pershin, Supersymmetric Born-Infeld from the pure spinor formalism of the open superstring, JHEP 0301 (2003) 023 [hep-th/0205154]; N. Berkovits, Multiloop Amplitudes and Vanishing Theorems using the Pure Spinor Formalism for the Superstring, JHEP 0409 (2004) 047 [hep-th/0406055]; N. Berkovits, Quantum Consistency of the Superstring in AdS $S_{5} \times S^{5}$ Background, hep-th/0411170.

[11] N. Berkovits and P. Howe, Ten-dimensional supergravity constraints from the pure spinor formalism for the superstring, Nucl. Phys. B635 (2002) 75 [hep-th/0112160].

[12] O. Chandia and B. C. Vallilo, Conformal invariance of the pure spinor superstring in a curved background, JHEP 0404 (2004) 041 [hep-th/0401226].

[13] J. de Boer and K. Skenderis, Covariant computation of the low energy effective ection of the heterotic superstring, Nucl. Phys. B481 (1996) 129 [hep-th/9608078].

[14] N. Berkovits, O. Chandia and V. Pershin, Gauge and Lorentz Anomalies in the Pure Spinor Formulation of the Heterotic Superstring, to appear. 